\title{
Evaluation of Residual Stress Effects in Aft Pressure Ring Frame of a Royal Australian Air Force P-3C Maritime Patrol Aircraft
}

\author{
Kevin Walker ${ }^{1, a^{*}}$, Michael Ryan ${ }^{1, b}$ and James Ayling ${ }^{2, c}$ \\ ${ }^{1}$ DST Group, 506 Lorimer St Fishermans Bend, Victoria, Australia \\ ${ }^{2}$ Airbus Group Australia Pacific, NSW, Australia \\ akevin.walker@dsto.defence.gov.au, bmichael.ryan@dsto.defence.gov.au, \\ james.ayling@airbus.com
}

\begin{abstract}
Keywords: X-Ray Diffraction, Finite Element Analysis, Thermal Control, P-3C Orion, Fuselage Pressure Frame
\end{abstract}

\begin{abstract}
Aft pressure bulkhead ring frames on the RAAF P-3C Orion aircraft are being replaced due to in-service fatigue cracking. Installation of the new 7075-T6511 extrusion L-section circular frame requires local adjustment of the included angle at various positions around the circumference. The adjustment should be carried out under a controlled temperature in the range of $135-160^{\circ} \mathrm{C}$. However in some recent cases the temperature was either not well controlled and/or was below that range, raising concerns about the potential for adverse residual stresses in a region already known to be susceptible to fatigue. This paper details an investigation including 3-D non-linear finite element (FE) modelling and X-Ray diffraction based residual stress measurements on representative frame segments. The FE modelling was performed with NASTRAN using 3-D solid elements. Plastic behaviour was modelled using a Ramberg-Osgood approach. The X-Ray diffraction was performed using a Proto iXRD Combo instrument with a Cobalt X-ray source and a rectangular aperture (1.0, 1.5 or $2.0 \times 5.0 \mathrm{~mm}$ depending on the location) and also electro-polishing for depth data. Permanent resultant angles of the frame segments were also measured using a "MarSurf CD 120" contact profiler. Typically low residual stresses were found for both the within and the below specification temperature cases. The FE and measurements were in reasonable agreement giving confidence in the results. The results from this work supported a decision to clear affected aircraft for a return to service.
\end{abstract}

\section{Introduction}

The aft pressure bulkhead structure at FS 1117 on the P-3 Orion aircraft, Figure 1, includes an approximately "L" shaped forward frame segment, Figure 2. The frame, manufactured from 7075T651 alloy extrusion has a nominal included angle of $100^{\circ}$. Due to cracking which has occurred in service at the location of interest shown in Figure 2, the frames are being replaced on some RAAF aircraft. During installation of the new frames, the included angle needs to be adjusted in some areas. In the upper region of the fuselage the angle is tightened to about $94^{\circ}$, and in the lower end the angle is increased to about $102^{\circ}$. The angle adjustments are performed mechanically as shown in Figure 3 with locally applied heating [1]. The temperature range for the heating should be carefully controlled and thermocouple measurements are to be taken to ensure that the temperature range is controlled to the range $135-160^{\circ} \mathrm{C}$. In some cases however, thermocouple measurements were not taken and it was thought that the hand forming in those cases may have occurred at a temperature below the specified range. 


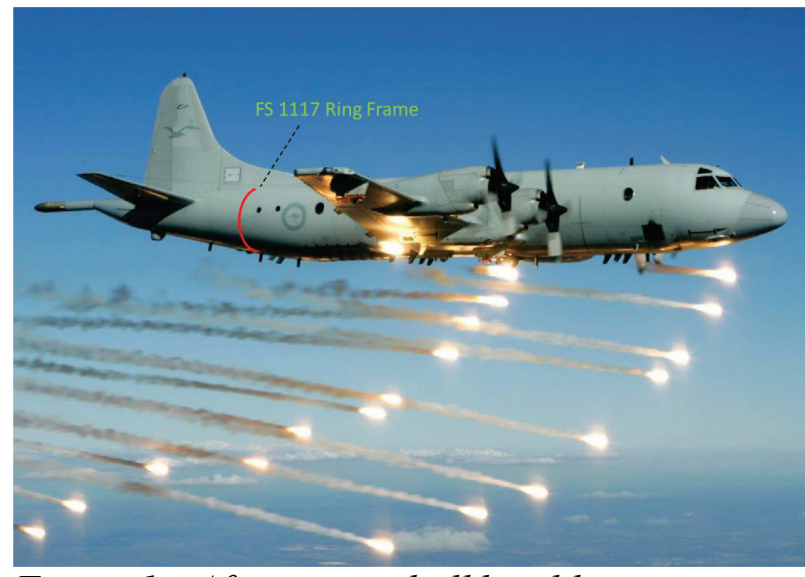

Figure 1: Aft pressure bulkhead location at FS 111, RAAF AP-3C aircraft

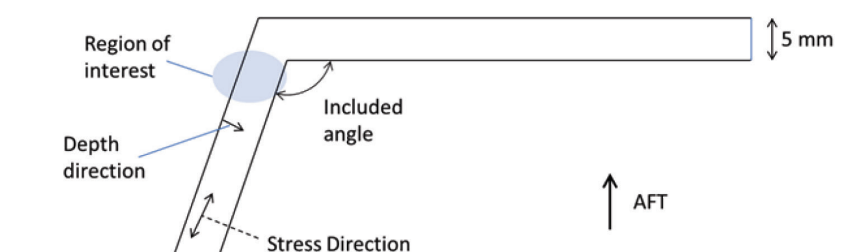

Figure 2: Cross section of forward ring frame of FS 1117 aft pressure bulkhead

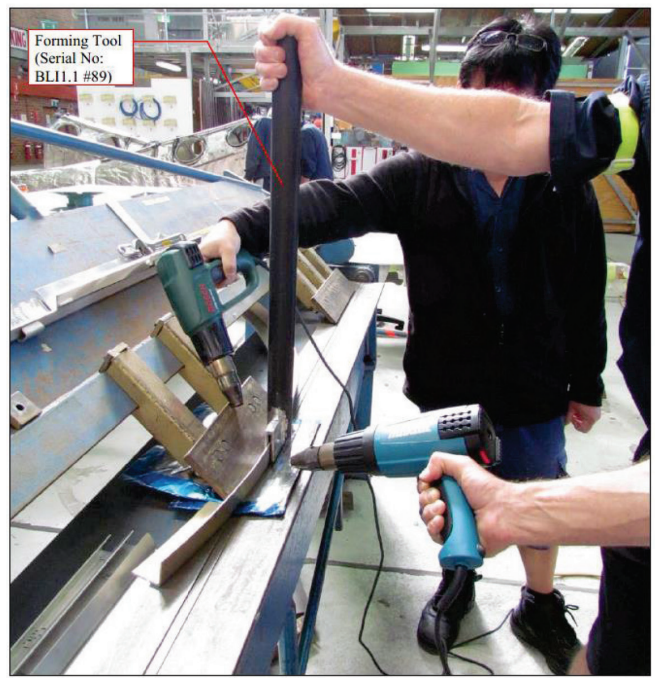

Figure 3 : Hand forming process for FS 117 forward ring frame included angle adjustment

Even if the forming process is carried out correctly, residual stresses are expected to be present in the frame. But there was a concern that if the process was carried out incorrectly in terms of temperature control in particular, then the final residual stress state might be such that fatigue crack growth and fatigue life would be significantly degraded.

This paper provides a summary of experimental and analytical activities to investigate this issue. The objective was to determine if performing the ring frame adjustments without correct temperature control would result in a significantly detrimental outcome in terms of residual stress. Samples of ring frame segments were obtained including the following scenarios; upper and lower locations, parts removed from a service aircraft, adjusted parts with and without proper temperature control, and nominal as manufactured parts. This resulted in a total of eight test specimens. The main area of concern is the upper frame where the nominal angle is reduced. That scenario is expected to result in tensile residual stress at the inner surface which is the location where fatigue cracking has been experienced in service.

X-Ray residual stress measurements were conducted using a Proto iXRD Combo with a cobalt Xray source and a rectangular aperture $(1.0,1.5$ or $2.0 \times 5.0 \mathrm{~mm})$. Measurements were taken wherever possible on the inner and outer surfaces at the location of interest, and also into the depth using electro-polishing. The analytical work included three-dimensional elastic-plastic finite element 
modelling using MSC Nastran. These results were compared and used to determine if performing the frame adjustments without controlling the temperature would result in a detrimental outcome in terms of residual stress.

\section{Finite Element Modelling}

Finite element modelling was performed using NASTRAN with six-sided, eight-noded solid nonlinear elements. Plastic behaviour was modelled using a Ramberg-Osgood approach. Further details are provided in [1]. The most significant result for comparison later with the measurements relates to the Specimen 1 scenario of an upper location with a nominal angle reduction of $6^{\circ}$. The FE predicted stress in that case is $385 \mathrm{MPa}$ with room temperature forming, and $300 \mathrm{MPa}$ with $160^{\circ} \mathrm{C}$ forming The FE analyses also determined that if the stress was tensile at the inner surface, then the stress is compressive at about the same magnitude at the outer surface. This finding was very important for the assessment of the X-Ray based residual stress measurements because many of them were conducted from the outer surface due to texturing and access difficulties with inner surface measurement. These were the key findings from the FE modelling to be compared with the X-Ray rsidual stress and final angle measurements which are discussed next.

\section{Measurement details}

Specimen descriptions. A total of eight test specimens were available. These covered both the upper and lower locations, combined with the three scenarios of forming with and without proper temperature control, and no forming at all (as manufactured). Another two were the upper and lower sections removed from a service aircraft, Tail Number A09-662. It was also noted whether the section was from the left or right hand side (LH, RH), but that was not expected to be a significant variable here and was not explicitly investigated.

The specimens provided have an arc length of about $500 \mathrm{~mm}$. A typical example is shown in Figure 4.

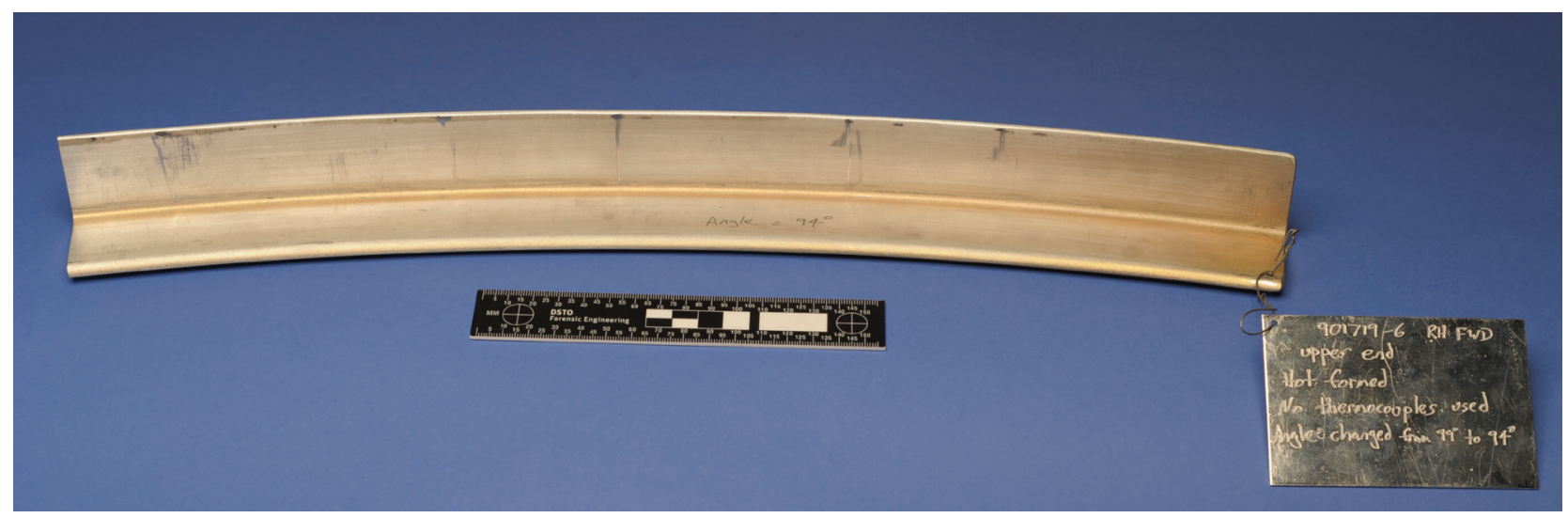

Figure 4 Typical FS1117 ring frame specimen provided for investigation

X-Ray diffraction details. X-Ray diffraction measurements were performed with a Proto iXRD Combo with a Cobalt X-Ray source and a rectangular aperture (1.0, 1.5 or 2.0 x $5.0 \mathrm{~mm})$. Profile measurements into the depth were made wherever possible. Depth measurements were performed by taking progressive electro-polishing cuts using the Proto system. Most measurements were taken from the outer surface, but some readings were also undertaken where possible on the inner surface. The typical set-up for an outer surface measurement is shown in Figure 5. 


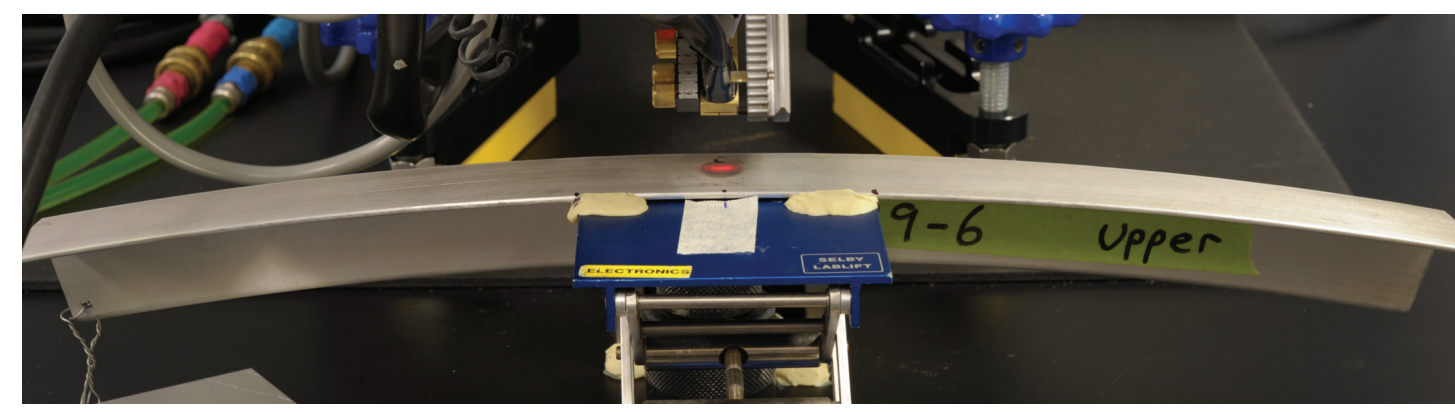

Figure 5 : Typical set-up for outer surface measurement

When measurements were taken on the inner surface it was necessary to cut away part of the thick flange to ensure that the X-Rays were not shielded from the region of interest. A sample with the flange cut away is shown in Figure 6

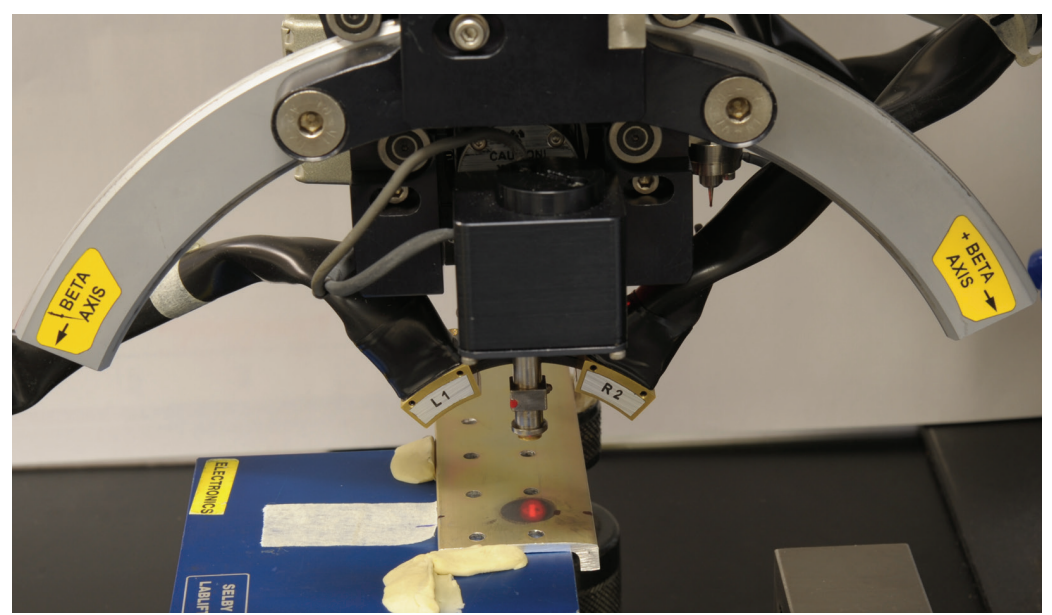

Figure $6:$ XRD set up detail showing case where thick flange is cut away to allow inner surface measurement

Included angle measurement details. The included angle measurements were performed using a Mahr "MarSurf CD 120" contact profiler. Measurements were taken at each end of each sample, and also at the mid-region. The precision of the instrument is such that angle measurement to at least the nearest $0.1^{\circ}$ or better is expected.

\section{Results}

The residual stress results into the depth from the outer surface are detailed in Figure 7. In addition, the following surface only results were obtained for the residual stress measurements:

\begin{tabular}{|l|l|}
\hline $\begin{array}{l}\text { Specimen } 8 \text { (lower frame, as manufactured) } \\
\text { Outer surface }\end{array}$ & $\begin{array}{l}\text { Specimen 3 (lower frame, removed from } \\
\text { Service aircraft) Inner Surface } \\
\text { Stress }-50+/-10 \mathrm{MPa}\end{array}$ \\
&
\end{tabular}




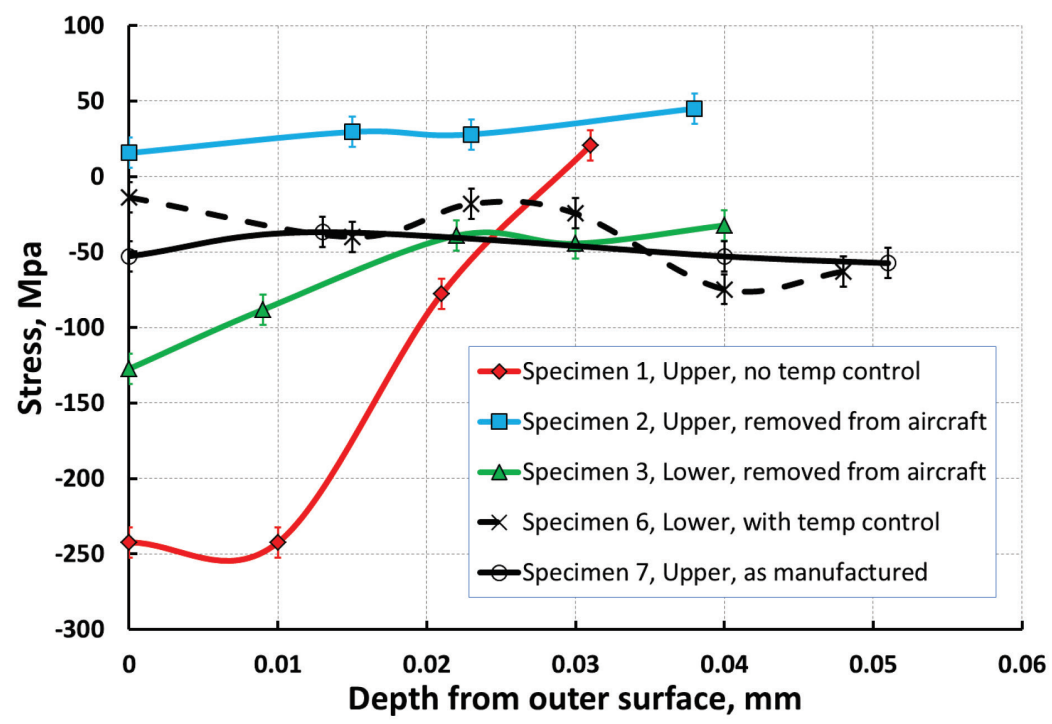

Figure 7 : X-Ray diffraction residual stress measurements with depth

The included angle measurements are detailed in Table 1.

Table 1: Included angle measurement results

\begin{tabular}{|c|c|c|c|c|c|c|c|}
\hline \multirow[t]{2}{*}{ Specimen \# } & \multirow[t]{2}{*}{ Description } & \multirow[t]{2}{*}{ Formed, Y/N } & \multirow{2}{*}{$\begin{array}{l}\text { Thermocouples } \\
\text { used, Y/N }\end{array}$} & \multirow{2}{*}{$\begin{array}{l}\text { Desired angle } \\
\text { change, } \\
\text { degrees }\end{array}$} & \multicolumn{3}{|c|}{ Measured Angle, degrees } \\
\hline & & & & & Tag end & Middle & Other end \\
\hline 1 & RH Upper & $\mathrm{Y}$ & $\mathrm{N}$ & 100 to 94 & 92.7 & 91.0 & 93.0 \\
\hline 2 & $\begin{array}{l}\text { RH Upper, } \\
\text { removed } \\
\text { from A09- } \\
662\end{array}$ & N/A & N/A & N/A & 95.1 & 94.4 & 94.1 \\
\hline 3 & $\begin{array}{c}\text { RH Lower, } \\
\text { removed } \\
\text { from A09- } \\
662\end{array}$ & N/A & $\mathrm{N} / \mathrm{A}$ & N/A & 102.2 & 101.8 & 103.1 \\
\hline 4 & RH Lower & $\mathrm{Y}$ & $\mathrm{N}$ & 100 to 102 & 102.8 & 104.3 & 103.2 \\
\hline 5 & LH Upper & $\mathrm{Y}$ & $\mathrm{Y}$ & 100 to 94 & 94.8 & 94.0 & 95.0 \\
\hline 6 & LH Lower & $\mathrm{Y}$ & $\mathrm{Y}$ & 100 to 102 & 102.6 & 103.1 & 104.7 \\
\hline 7 & RH Upper & $\mathrm{N}$ & N/A & None & 98.6 & 98.7 & 98.0 \\
\hline 8 & RH Lower & $\mathrm{N}$ & N/A & None & 98.4 & 98.2 & 97.2 \\
\hline
\end{tabular}

\section{Discussion}

The objective of this work was to measure the state of residual stress and final included angle for a range of representative scenarios in P-3 aft pressure bulkhead forward ring frames. It was not possible to obtain residual stress measurements in all the samples. This was attributed to texture effects in the elongated "pancake" type grain structure which is present in the 7075-T651 extruded ring frame segments. The texturing makes it more challenging for the X-Ray approach to obtain acceptable diffraction signal to determine the stress condition.

The peak residual stress in the tested ring frames were found to be mostly low, i.e. less than 50 $\mathrm{MPa}$ in magnitude. The most significant residual stress measured was about -250 MPa (compression) at the outer surface on sample 1 which was at the RH upper location (no temperature control) with a nominal angle change from $100^{\circ}$ to $94^{\circ}$. In comparison, the $\mathrm{FE}$ result for a nominal 
$6^{\circ}$ angle change is $-385 \mathrm{MPa}$ (compression) at room temperature, and $-300 \mathrm{MPa}$ at $160^{\circ} \mathrm{C}$. Given that some heat was applied to Specimen 1, the comparison between the FE and the measurement is considered reasonable, with the FE being more conservative. In this case, the "spring back" effect explains why the stress state would be significant compression on the outer surface. The measured final angle was in the range $91-93^{\circ}$ (see Table 1), which is slightly lower than the "target" angle of $94^{\circ}$. So the residual stress is expected to be larger in magnitude than if the final angle was higher (less change). These results can be compared with Specimen 2 which was removed from a service aircraft. The upper location nominally requires a reduction in the angle from 100 to $94^{\circ}$. For Specimen 2, the measured angle was about $94-95^{\circ}$ which implies that less change was applied compared to Specimen 1. The peak residual stress at the outer surface for Specimen 2 was very low, $15 \mathrm{MPa}$, which is consistent with the observations just described.

Specimen 3 was removed from an aircraft and was at the lower location, where the angle is nominally increased from 100 to $102^{\circ}$. The "spring back" effect mentioned earlier suggests that a state of residual tension would be expected at the outer surface, but in fact the measured stress was about $-13.0 \mathrm{MPa}$ (compression), which is very low. The final angle was measured at about $102-103^{\circ}$, which is very close to the target value. But the angle change in this case was likely very small.

The highest absolute stress measured in any sample was a tensile stress close to $+50 \mathrm{MPa}$ at a depth of about $0.04 \mathrm{~mm}$ in Specimen 2, which was removed from the upper location in a service aircraft A09-662. The final angle was measured to be 94-95 . Being the upper location, like Specimen 1, the nominal condition would be decreasing the angle from 100 to $94^{\circ}$. In this case it appears likely that the angle change was therefore in the range $5-6^{\circ}$, which is less than for Specimen 1 where the nominal change was $7-9^{\circ}$. It is therefore consistent that Specimen 1 would exhibit larger magnitude residual stress than Specimen 2.

It is important to note that the measured residual stress even in new ring frame segments with no angle change were around -50 MPa (compression) at the outer surface (Specimens 7 and 8). This is a measure of the typical residual stress in the as-manufactured condition, so this gives an indication of the possible starting point for residual stress in these frames.

\section{Conclusions}

The residual stress state and final angle for a range of representative FS1117 forward ring frame samples were measured and reported. The residual stresses were generally low. The results suggest that the residual stress state is more likely to be related to a combination of the initial state and the angle change itself rather than whether the temperature was controlled or not. The FE analysis showed that although the temperature condition had some effect, the main parameter which influenced the resiudal stress condition was the permanent angle change. The X-Ray measurements showed that the FE analysis was conservative. It is therefore concluded that the ring frame segments where poor temperature control was applied would be expected to be not significantly worse in terms of residual stress than the cases where the specified temperature range was applied. This supported a decision to allow the affected aircraft to be returned to service.

\section{Acknowledgements}

The authors appreciate the assistance of Mr Bruce Grigson, DST Group, who conducted the included angle measurements using the Mahr system.

\section{References}

1. Scholz, L., FS1117 residual stress investigation and analysis, A09-ER-003002, 2015, Airbus Group Asia Pacific. 